\title{
The Relationship Between Collectivism and Seeking Product-Related Information on Social Networking Sites: An Abstract
}

\author{
Todd Pezzuti and James M. Leonhardt
}

\begin{abstract}
Social networking sites provide consumers with the opportunity to easily and rapidly obtain information about brands, products, and services. Considering the effect that online word-of-mouth can have on important marketing outcomes, such as product sales and brand loyalty, it is important to understand the factors that drive consumers to seek product-related information on social networking sites. The present research focuses on whether and how cultural collectivism influences the extent that consumers seek product-related information through discussion with others on social networking sites. Study 1 uses archival data which includes the collectivism score for 53 different countries and the extent that consumers in those countries seek product-related information on social networking sites. The results suggest that collectivism exerts a positive effect on the extent that consumers seek product-related information on social networking sites. The results of a second study using primary data suggest that the positive effect of collectivism on information seeking is mediated by trust in the members of social networking sites.
\end{abstract}

\footnotetext{
T. Pezzuti $(\bowtie)$

Universidad de Chile, Santiago, Chile

e-mail: tpezzuti@gmail.com

J. M. Leonhardt

University of Nevada, Reno, NV, USA

e-mail: jleonhardt@unr.edu
} 